Review

\title{
Calpain 3, the "gatekeeper" of proper sarcomere assembly, turnover and maintenance
}

\author{
Jacques S. Beckmann ${ }^{\mathrm{a}, *}$, Melissa Spencer ${ }^{\mathrm{b}}$

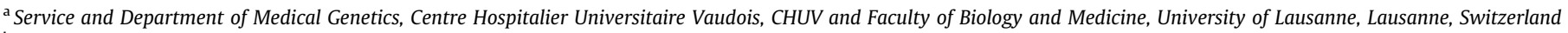 \\ ${ }^{\mathrm{b}}$ Department of Neurology, University of California Los Angeles, David Geffen School of Medicine at UCLA, 635 Young Dr. South, Los Angeles, CA 90095-7334, USA
}

\section{A R T I C L E I N F O}

\section{Article history:}

Received 14 April 2008

Received in revised form 18 August 2008

Accepted 27 August 2008

\section{Keywords: \\ Calpain \\ Muscle \\ Dystrophy \\ LGMD \\ Limb girdle \\ Sarcomere \\ Atrophy \\ Titin}

\begin{abstract}
A B S T R A C T
Calpain 3 is a member of the calpain family of calcium-dependent intracellular proteases. Thirteen years ago it was discovered that mutations in calpain 3 (CAPN3) result in an autosomal recessive and progressive form of limb girdle muscular dystrophy called limb girdle muscular dystrophy type 2A. While calpain 3 mRNA is expressed at high levels in muscle and appears to have some role in developmental processes, muscles of patients and mice lacking calpain 3 still form apparently normal muscle during prenatal development; thus, a functional calpain 3 protease is not mandatory for muscle to form in vivo but it is a pre-requisite for muscle to remain healthy. Despite intensive research in this field, the physiological substrates of the calpain 3 protein (hereafter referred to as CAPN3) and its alternatively spliced isoforms remain elusive. The existence of these multiple isoforms complicates the search for the physiological functions of CAPN3 and its pathophysiological role. In this review, we summarize the genetic and biochemical evidence that point to loss of function of the full-length isoform of CAPN3, also known as p94, as the pathogenic isoform. We also argue that its natural substrates must reside in its proximity within the sarcomere where it is stored in an inactive state anchored to titin. We further propose that CAPN3 has many attributes that make it ideally suited as a sensor of sarcomeric integrity and function, involved in its repair and maintenance. Loss of these CAPN3-mediated activities can explain the "progressive" development of muscular dystrophy.
\end{abstract}

(c) 2008 Elsevier B.V. All rights reserved.

\section{Introduction LGMD history}

Limb girdle muscular dystrophy (LGMD) is a group of progressive muscle diseases, with weakness and wasting affecting predominantly shoulder and pelvic girdle muscles of both males and females with clinical onset, in general, in the first three decades [1-4]. The initial clinical description of what is now known as an LGMD goes back over 100 years when a German neurologist, Wilhelm Neurich Erb, described a juvenile form of scapulo-humeral form of muscular dystrophy [5]. In 1954, the term "Limb Girdle Muscular Dystrophy” was coined [6], yet since its description and until recently, it has been plagued by uncertainties and discreditation [7-9].

While Chung and Morton and Jackson and Carey each reported an autosomal recessive form of LGMD as early as 1959 [10,11], the first reports of an autosomal dominant inheritance go back to 1986 $[12,13]$. Prompted by the early successes in what later became known as "positional cloning", the dominant and recessive forms were defined, respectively, as LGMD1 (autosomal dominant inheritance) and LGMD2 (autosomal recessive inheritance) with

\footnotetext{
* Corresponding author.

E-mail address: jacques.beckmann@chuv.ch (J.S. Beckmann).
}

LGMD2A (OMIM \#253600) being the first for which a chromosomal locus was assigned [14].

Since then close to 20 LGMD loci have been uncovered and often identified [3,15-18]. Interestingly the majority of LGMD genes encode for structural proteins. For example, mutations in several transmembrane, cytoskeletal proteins have been identified such as dysferlin (LGMD2B; OMIM \#253601), the four sarcoglycans $(\alpha-, \quad \gamma-, \quad \beta-$ or $\delta$-sarcoglycan; LGMD2C-F; OMIM \#253700, \#608113, \#604286 and \#601287, respectively) and caveolin 3 (LGMD1C; OMIM \#607801). Mutations were also identified in other structural proteins linked to the cytoskeleton of the sarcomere including telethonin (LGMD2G; OMIM \#601954), titin (LGMD2J; OMIM \#608807) and myotilin (LGMD1A; OMIM \#159000). Finally, mutations in a nuclear protein (lamin A/C (LGMD1B; OMIM \#159001) were linked to a form of autosomal dominant LGMD.

The gene involved in LGMD2A encodes for CAPN3, a member of the calpain family, a family of said non-lysosomal, soluble cytosolic calcium-dependent proteases. The name "calpain" was derived by contraction of calcium and papain [19], in reference to the requirement for calcium and the sequence homology between this other family of proteases. The calpains were shown to manifest ubiquitous or tissue-specific expression patterns and have been the sub- 
ject of numerous studies (over 4900 different PubMed entries by end of April 2008) with remarkable progress reported in the fourth FASEB Summer Research Conference on the biology of Calpains in Health and Disease (July 2007). Most members of this gene family are thought to be regulatory or "noble" proteases that do not completely demolish their substrates but cleave them in a controlled and limited manner, thereby modulating their function in an irreversible fashion. A wide variety of proteins can be cleaved in vitro by calpains, and finding their proper physiological substrates is, to say the least, not an easy task. This situation prevails also for CAPN3 as one is, almost 20 years after its initial description [20], still in search of its natural physiological substrates (e.g. [21]). Noteworthy, CAPN3 is nevertheless the first and still the only gene product of this gene family that was demonstrated to be directly and causally involved in the etiology of an inherited disease. Moreover, with predominant expression in skeletal muscles [20,22], CAPN3 is the first enzyme to be incriminated in a muscular dystrophy (or specifically in a LGMD). Since then others have joined this list, such as the E3 ubiquitin ligase Trim32 (LGMD2H; OMIM \#254110, [23]), the glycosyltransferases FKRP (LGMD2I; OMIM \#607155, [24]) or Fukutin (LGMD2M; OMIM \#611588, [25,26]), or the known or putative protein-O-mannosyl-transferase-1 POMT1 (LGMD2K; OMIM \#609308, [27]), or O-mannosyl-transferase-2 (OMIM $* 607439$, coined LGMD2N in [28]). In this report, we shall discuss CAPN3 in relation to its pathologic and etiologic role in LGMD2A. Some of the ideas presented here are common to those of Kramerova et al. [29] and Duguez et al. [30]. Additional supportive data was recently published by Chen et al. [31].

\section{What have we learned from the genetics?}

The average overall prevalence for all of the AR forms of LGMD is estimated to range from $1: 15,000$ to $1: 100,000$, and this can vary extensively between populations [2,32-36], and among these LGMD2A seems to be the most frequent in defined populations $[3,33,35,37,38]$, making it the second most frequent autosomal recessive muscle disease in northeastern Italy after spinal muscular atrophy [37]. Unlike for cystic fibrosis, there are no particular mutational CAPN3 hot spots, though some mutations may be more frequent than others, often reflecting ancient founder effects in consanguineous isolates - new private mutations appear constantly - so that the list of pathogenic variants increases steadily (see http://www.dmd.nl/CAPN3, version 080404 for a complete listing; [39]).

Some 440 distinct CAPN3 mutations are known to date (see http://www.dmd.nl/CAPN3), of which 212 are non-synonymous amino acid changes, and at least 135 gene-inactivating mutations resulting, most commonly, in nonsense mediated decay (NMD). The recognition of LGMD2A patients whose skeletal muscles are devoid of CAPN3, as they carry two gene-inactivating alleles, allowed one to infer that a lack of CAPN3 (the protease and/or other functional properties) is pathogenic as it is indispensable for the maintenance and survival of functional skeletal muscles [40,41]. Pathogenicity was also shown to be associated with loss of "proper proteolytic activity" as some mutations have been shown to reduce the ability of CAPN3 to proteolyze potential substrates such as fodrin and HSP60 or to autolytically self-cleave [42-44]. However, loss of enzymatic activity is not the case for some pathogenic LGMD2A missense mutations and therefore, it suggests that the latter may simply prevent substrate specificity or that CAPN3 may perform other as yet unidentified non-proteolytic roles (e.g. [45-47]).

The distribution of these LGMD2A mutations along the gene is also informative. The CAPN3 gene spans 24 exons spread over 53 (Ensembl ENSG00000092529) to 65 kilobases of genomic DNA
(NCBI NC_000015.8) and encodes for a 3316 basepairs long mature mRNA (Ensembl ENST00000318023; NCBI NM_000070) directing the synthesis of CAPN3 also known as p94, an 821 amino acid long full-length protein of $94 \mathrm{kDa}$ possessing three characteristic unique regions (Fig. 1B): a proline-rich NS domain ( $\mathrm{N}$-terminal sequence), IS1 and IS2 domains (inserted sequences 1 and 2), with IS2 containing a potential nuclear localization signal (NLS) [20]. Obviously gene invalidating mutations (e.g. frameshift mutations) can fall anywhere along this gene's coding exons. It is, in contrast, informative to consider the distribution of non-synonymous missense mutations along the CAPN3 polypeptide or 3D structure [46]. One can see, upon plotting this distribution (along a scanning window of $20 \mathrm{aa}$ ), that defined structural regions (in particular, those associated with the active site of the molecule) seem to have a higher rate of mutation than others, though at this level of resolution, mutations seem to present over the entire length of the protein (Fig. 1A, see also [39]). Thus, domains IIa and IIb are mutationrich regions, while in contrast, the CAPN3-specific sequences IS1 and IS2, encoded, respectively, by exon 6 and exons 15 and 16, seem relatively poor but not free of such mutations. Of interest, these unique domains are also not necessary for activation as the conventional calpains that lack these sequences, still retain activity, albeit at a higher calcium concentration. This is in line with the fact that these elements are phylogenetically less conserved than the rest of the gene: in a 9 species comparison (bovine, dog, horse, human, macaque, mouse, pig, rat and sheep) of full-length CAPN3 consensus sequence, there are, respectively, 26/62, 16/63 and 26/76 amino acid substitutions in NS, IS1 or IS2, of which 22, 11 and 23 are non-conservative substitutions, often present as clusters. This is to be contrasted to 68 changed residues, of which 29 are non-conserved, for the rest of the protein, i.e., the remaining 620 amino acid residues. These CAPN3-specific domains may thus tolerate numerous changes without harmful consequences.

At least 10 distinct CAPN3 transcriptional and post-transcriptional isoforms have been described [22,48-50], with splicing isoforms involving mostly exons 6,15 or 16 and thus the corresponding proteins may lack or contain either or both IS1 and IS2. These isoforms that lack IS1 and IS2 appear to be primarily important during development and muscle regeneration as they are not expressed in healthy adult muscle.

Human CAPN3 differs in at least one respect from that of rodent in that yet another lens-specific isoform is seen only in rodents which makes use of an alternative promoter that has been lost during evolution in the human lineage [51], and which leads to the synthesis of a protein that has a different $\mathrm{N}$-terminal peptide lacking the NS domain $[50,52,53]$. The existence of these various isoforms complicates the search for the physiological functions of CAPN3 as one no longer needs to reason in terms of a single gene product but of a multiplicity thereof, a difficulty further compounded by the possibility of post-translational modifications including phosphorylation (D. Goll, personal communication) and/or tissue-specificities [22,48-50,54,55]. Thus, with so many isoforms, is there a particular one that plays a central causal role in the etiology of LGMD2A? As will be seen further in this review, genetic evidence allows the designation of one particular suspect.

The fact that IS1 and IS2 [34,45,56] also harbor pathogenic mutations (including null-type mutations) suggests that it is the mature full-length product of CAPN3 (i.e., p94 as it was initially named by its discoverers [20]) that constitutes the (main) etiologic target of LGMD2A: indeed, these mutations are not likely to affect the alternatively spliced isoforms lacking these particular sequences, leaving only mature full-length CAPN3 as the defective entity. This is further corroborated by the observation that (i) while the alternatively spliced isoforms are synthesized during development and muscle maturation, they are absent in fully differentiated muscles and (ii) that LGMD2A patients or animal models 
A Positions with substitutions per bin of 20 amino acids

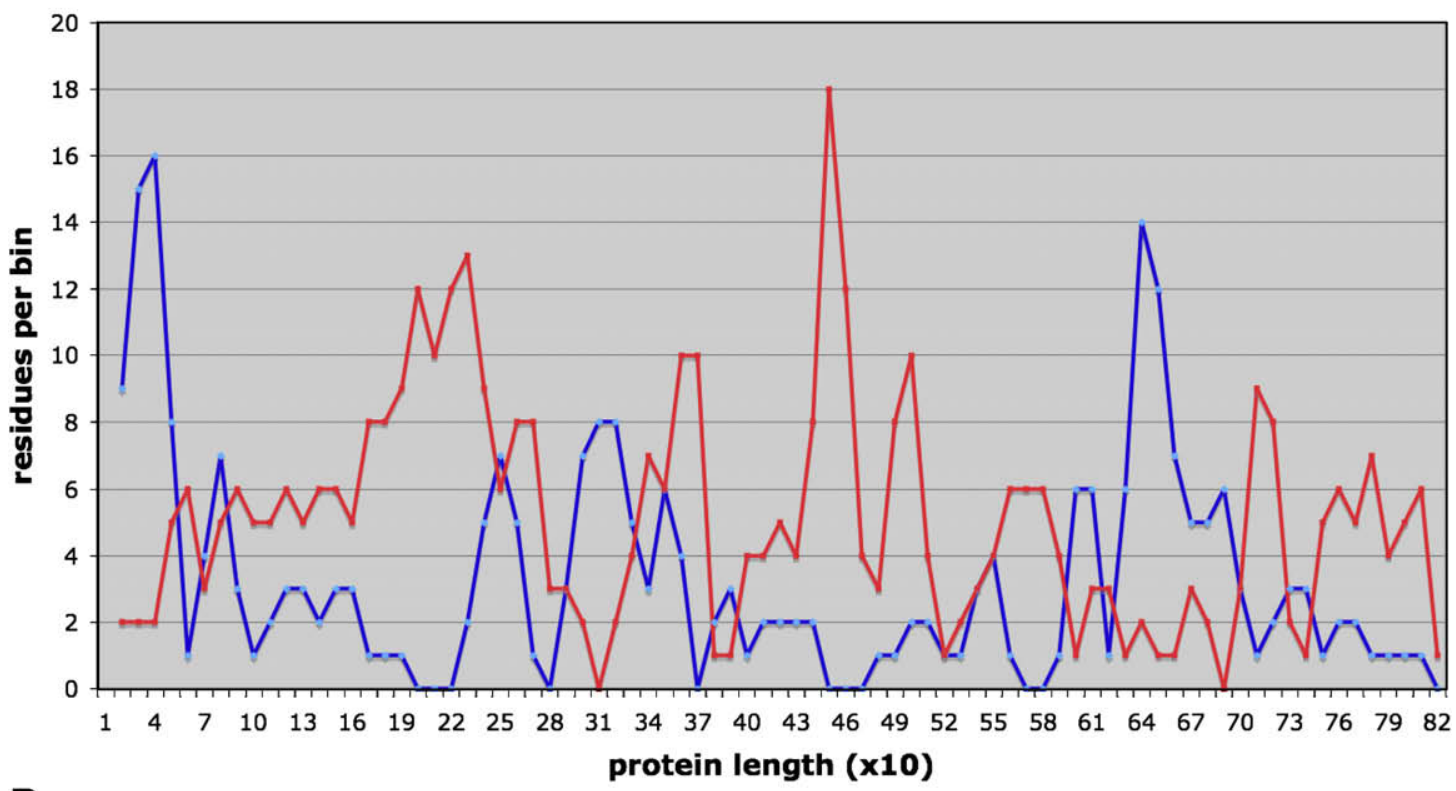

B

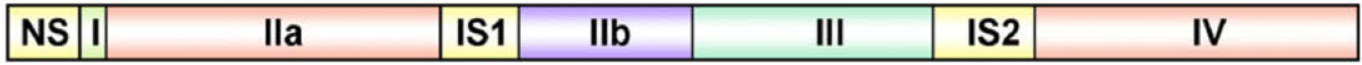

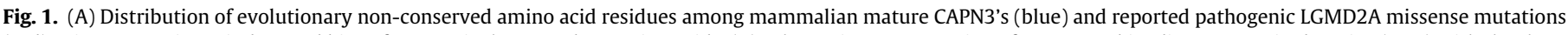

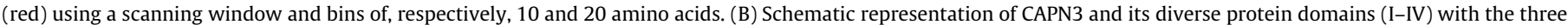
calpain 3-specific domains, NS, IS1 and IS2.

with obliterated calpain 3 genes, are born with apparently normally constituted muscles, yet the disease settles in progressively as they grow and age.

\section{What have we learned from biochemical or structural analyses of the calpains?}

We shall not detail here all of CAPN3's known properties, as the calpains in general, and CAPN3 in particular are discussed in numerous reviews (e.g. [39,57-61]). We will however highlight features relevant for the pathophysiological model proposed herein.

Besides its three unique peptides (NS, IS1 and IS2) and tissuespecificity, a number of other distinctive features distinguish CAPN3 from most of the remaining calpains. Like the ubiquitous calpains, CAPN3 does bind calcium ions [62,63], but unlike the former, it works at physiological, submicromolar calcium concentrations, it turns over rapidly and proteolytic activity of CAPN3 is not inhibited by the ubiquitously expressed protein calpastatin (the endogenous inhibitor of calpains, which can also be cleaved by calpain 3; for review see [64]), nor does it form heterodimer complexes with the small calpain 4 subunit (e.g. [57-59]). Instead gel-filtration [65] and X-ray diffraction [66] studies suggest that it seems to form stable homodimers with its catalytic centers falling on opposite ends of the dimer.

Functional and structural studies enabled the delineation of distinct protein structural domains, which have been reviewed elsewhere $[39,46,57,58]$ as well as the mapping and identification of its inter- and intramolecular autolytic cleavage sites [63,67-69]. From these studies, the activation mechanism has been inferred.

Purified CAPN3 has a very short half-life of less than $10 \mathrm{~min}$, and is rapidly degraded through autolysis [70]. Yet it was shown to be stable within intact, dissected muscle biopsies $[71,72]$ or myofibrillar fractions [65]. This latter finding is likely due to the necessity for CAPN3 to be anchored to the cytoskeleton of the sarcomere to prevent its degradation through autolysis or by other cellular proteases.

\subsection{The titin connection}

While CAPN3 undoubtedly resides in diverse cellular locations, a preponderance of evidence suggests that the majority of CAPN3 is anchored to the cytoskeleton of the skeletal muscle sarcomere. Both yeast-two hybrid and immunohistological data suggest that mature full-length CAPN3 is stored within the sarcomere bound to the gigantic ( $1 \mu \mathrm{m}$ long), elastic and highly modular scaffold protein, titin (also known as connectin). Titin acts both as a molecular ruler and blueprint for sarcomere assembly, spanning half a sarcomere from Z-disk to M-line, and as an adjustable molecular spring central to mechano-transduction of the contracting myofiber (e.g. [73,74]). Noteworthy, both with titin itself (LGMD2J), and among the many sarcomeric proteins known to bind directly or indirectly to titin, one can find additional members of the LGMD family such as telethonin (LGMD2G; OMIM \#601954) or myotilin (LGMD1A, OMIM \#159000). Binding to mature full-length CAPN3 is mediated through titin's N2A and M-line specific sequences $[68,69,75,76]$. These in vitro data were confirmed by protein binding studies [77] and immunohistochemistry that also showed localization at the Z-line $[69,75,78]$.

Titin, the third most abundant protein in muscle [79], and its mRNAs are widely more profuse than those of calpain 3 [80]. Hence, it is likely that titin is present within the sarcomere in vast stoichiometric excess with respect to CAPN3; and as such can serve as a reservoir for the latter, which would be dispersed and stored in this dense lattice structure in a presumably inactive form. Ojima et al. [81] further showed that full-length mature CAPN3 is incorporated into myofibrils via titin only once the sarcomere structures are fully mature. It was suggested that titin binding, through sequences within IS2, overlap with its putative nuclear localization signal (NLS) that may stabilize CAPN3 and 
protect it from rapid autolysis within IS1 $[65,68]$, and that this cleavage step is required for activation of its function $[68,69,82]$. Titin binding might thus function in conjunction with IS1 (the internal auto-inhibitory propeptide) to block the active site of mature CAPN3 from substrates and inhibitors [82], and perhaps to assume a role analogous to that of calpastatin, the known physiological inhibitor of the ubiquitous calpains (for review see [64]). Interestingly, the excision of IS2 makes CAPN3 more stable [76], a finding that may point to a "dual" role for IS2, an inhibitor of activity at rest and once it is activated, a factor that contributes to its lability.

Genetic evidence supports this CAPN3-titin connection. Titin mutations at the $\mathrm{M}$ line region of titin, near the CAPN3 binding sites were shown, when present in a homozygous state, to result in pathogenic conditions akin to LGMD as well as to a secondary CAPN3 depletion in patients with tibial muscular dystrophy (TMD or LGMD2J; OMIM \#608807) [83,84]. In mice, mutations in titin's N2 line alter CAPN3's ability to bind to it. This mutation results in recessive muscular dystrophy with myositis (mdm) and a secondary reduction of CAPN3 [85], though the pathogenic significance of this secondary CAPN3 defect in the mdm-mouse is still unclear. To examine the role of CAPN3 in mdm disease and to better understand the relationship between CAPN3 and titin, Huebsch et al. crossed the CAPN3 knock-out and CAPN3 overexpressing mouse to the mdm mouse. In these studies it was demonstrated that when the mdm mutation was placed on the CAPN3 knock-out background, it did not result in any change in the progression or severity of the mdm disease; in other words, lack of CAPN3 when its titin binding sites have been eradicated does not cause any additional phenotypic deterioration to animals bearing titin mutations. In contrast, overexpression of CAPN3 on the mdm background, exacerbated the mdm disease [86], presumably due to a loss of titin's ability to buffer CAPN3. This conclusion was derived from the knowledge that overexpression of CAPN3 on a wild type background bears no phenotype [87]. A similar condition caused by a C-terminal $\mathrm{M}$-line titin deletion resulting in a myopathy with secondary calpain 3 depletion and sarcomere disassembly was recently described in humans $[84,88]$. Thus, these data support the notion that too much free calpain 3 that is not anchored to titin can be toxic, and that healthy muscle fibers usually contain excess titin binding sites to anchor and regulate CAPN3. When loss of these binding sites occurs through genetic mutation, CAPN3 can be destabilized and the presence of excess CAPN3 can be toxic to the cell. All these elements point to an active and essential interaction between titin and CAPN3. Given the fact that the latter is known to exist as a homodimer and considering the general topography of the sarcomere, one still needs to determine how these homodimers bind to titin: for instance, are the two potential binding sites (one per calpain polypeptide chain) both utilized, are they bound to a single or to two distinct molecules, e.g. two adjacent titin chains and if so onto similar sites?

The high autolytic vulnerability and short half-life of pure CAPN3 poses another unanswered question, namely what stabilizes the nascent protein during translation on the polysomes? Could it be that posttranslational modifications may regulate the activated structure, or that the nascent polypeptide initially folds into an inactive conformation, and that external factors such as chaperones or perhaps one of its interactors are required to allow it to assume its active structure? Another possibility may be that translation of CAPN3 mRNA in muscle fibers is carried out on polysomes that are in close proximity to the sarcomere, where free titin- (or other as yet unknown) binding sites are present and available for the timely trapping and protection of these nascent protease polypeptide chains as they are being synthesized.

\subsection{Titin, a homeostatic regulator of CAPN3}

It is fascinating to ponder how the skeletal muscle fiber protects itself from unwanted potential damaging effects from this essential protease, which sits there like a Trojan horse, hidden and protected within the highly structured myofibrillar sarcomeres. If its activity is not carefully controlled, it can become a "conveyor of doom" in the form of unregulated proteolysis; however, it is interesting to note that if CAPN3 is ablated, one ends with a diseased muscle. Thus, it is no surprise that the skeletal muscles evolved a high buffering capacity against this delicate but powerful protease, to preserve robust homeostasis, tolerating and coping with large cellular differences in CAPN3 levels [87].

On one end, assuming that carriers of one null allele effectively have half the normal amount of CAPN3, this nevertheless leads to no major consequences as heterozygous individuals having but one active allele are asymptomatic. Moreover, considering both the recessive nature of inheritance and the fact that CAPN3 exists as a homodimer $[65,66]$ (assuming an equal contribution of the paternal and maternal alleles in any given fiber), one expects to find in healthy heterozygotes (carrying both a non-synonymous pathological mutation and a normal allele), the following three populations of dimeric mature full-length CAPN3 in, respectively, a 1:2:1 ratio: homodimers of the products of the healthy allele, heterodimers consisting, respectively, of the products of one mutant and one wild type allele, and homodimers of the products of the mutant allele. Mutant homodimers will be non-functional, though eventually also digested, inter-molecularly, by the remaining active enzyme. Compound heterodimers, in theory, may or may not be functional, as one might expect occasionally particular mutations to manifest a dominant negative effect inactivating the normal function of the heterodimer $[18,89]$. The fact that no dominant LGMD2A allele was described suggests that even under the latter scenario, there remains enough functional (wild type) homodimers to fulfill its essential cellular roles. Thus, even one quarter of the amount of normally present functional CAPN3 is likely to be fully compatible with regular muscle function.

On the other end, excess production of CAPN3 in heterozygous mdm mice (having one wild type and one mutant titin allele) or in transgenic mice overexpressing this protease is also non-pathogenic [87]. This can be explained by at least two factors. There exists a vast reservoir of titin binding sites to sequester and inactivate the excess CAPN3 $[79,86]$ : given the stoichiometric excess of titin over CAPN3 molecules [80], there are potentially many more binding sites than actually covered. To this, we can add the rapid autolytic cleavage of any residual free protease. If this hypothesis is correct, it implies that the myofibrillar system is extremely well buffered for both low (at least one quarter of the normal quantity) and excess amounts of functional CAPN3. This robustness raises hopes that a misadjusted or even partial therapeutic correction may nevertheless yield beneficial results.

CAPN3 was also observed in other cellular compartments besides the sarcomere $[90,91]$ such as the subsarcolemmnal membrane, where it can crosstalk with dysferlin [92,93] and cleave $\beta$ catenin [91], or the nucleus, to which it could be mobilized through its NLS signal in IS2 $[20,90]$. The interactions with the dysferlin protein complex may also involve AHNAK, a $700 \mathrm{kDa}$ scaffold protein that is also a potential CAPN3 substrate [94].

As is the case with CAPN3 within the myofibrillar lattice, it is necessary to properly regulate its proteolytic activity at these cellular sites, to avoid any unwanted deleterious havoc. By analogy, with the necessity to ensure proper spatio-temporal regulated control of CAPN3 activity and the fact that this protease is unaffected by calpastatin, one can surmise that other titin-like factors must act to regulate cytosolic, nuclear and membrane-bound CAPN3 as well. The identity of these postulated molecules is still unknown. Given, how- 
ever, that the main cellular CAPN3 reservoir is within the sarcomere, we shall confine our discussion to its role within this structure.

\section{Physiological role of CAPN3}

Numerous studies have been launched to identify substrates of CAPN3. These shall not be reviewed here. Our focus is to try to infer from current knowledge of this protease, of its known interactors and of the sarcomere, where in the muscle fiber one needs to look for these substrates.

Can one attempt, in view of the fact that CAPN3 sits predominantly within the sarcomere, to reconstruct its possible physiological function and role? First of all, a word of caution is needed. It may be hard to consider a single role for such an essential protein, even considering, as we do here, exclusively its mature full-length isoform. This is further substantiated by the observation that pathogenesis is observed with both loss and preservation of its proteolytic activity, thus suggesting a complex role for this protein. Second, numerous arguments suggest a role for CAPN3 in the maintenance of muscle integrity and function rather than in its ontogeny, development and maturation: CAPN3-deficient patients or animal models $[2,3,77,95]$ are born with apparently normal looking and functional muscles; full-length CAPN3 expression is a relatively late event during muscle development [51]; and its assembly into the myofibrils occurs after the sarcomere structures are fully mature [81]. And yet, CAPN3 appears to also play some role in development by downregulating membrane-bound $\beta$-catenin, as suggested by in vitro and in vivo studies [91]. This cellular function of CAPN3 is not essential for muscle formation, but it does impact one phenotypic feature of CAPN3 knock-out muscles: the nuclear/cytosolic ratio of skeletal muscle fibers [91]. This developmental role is also compatible with the observation that transgenic mice constitutively overexpressing alternatively spliced embryonic isoforms of CAPN3 (devoid of either exon 6 or 15), are severely affected, presenting with immature muscles resulting from a developmental block [87]. These are just examples of specialized, subcellular roles for CAPN3 or its isoforms, the lack of which can probably be compensated by redundant proteases that are active during development. Future investigations are likely to uncover many more of these specialized roles for CAPN3.

Thus, CAPN3 is tightly associated, in an inactive or autoproteolytically protected form with titin, in the middle of a tightly packed bundle of myofibrillar proteins where Brownian movement for proteins the size of CAPN3 is greatly limited [96,97]. From this we can infer that for a potentially labile enzyme that needs to be mobilized inside the muscle fibers in order to find its natural target substrates, that the latter cannot be far away from where this protease is anchored in a stable manner, i.e., within the sarcomere, as no other (small or large) stable reservoir for CAPN3 has currently been identified. To sum up thus far, the anchoring to titin has a triple function: (1) to keep CAPN3 from autolytically degrading itself and (2) to maintain it in a proteolytically inactive state, but readily turned on, and (3) to place it in proximity of its substrates.

We propose that one physiological role for CAPN3 is regulation of sarcomeric protein turnover and maintenance of structural integrity of sarcomere structure, in a reactive rather than active manner. Accordingly, as muscle activity can be damaging (e.g. [98]), one of mature full-length CAPN3's main roles may be to rid the cell of damaged sarcomeric proteins and in this way contribute to the maintenance of an effectively functioning muscle. The sarcomere is composed of the largest polypeptides in the world. These large proteins constitute, together with other abundant proteins such as muscle actin and myosin (and other sarcomeric proteins), a substantial fraction of total muscle protein in an extract. CAPN3 was shown to be able to cleave many of these sarcomeric proteins, both myofibrillar and cytoskeletal types. Fanin et al. for instance, reported nebulin degradation in healthy control muscle but not in muscles of any of eight LGMD2A patients tested [42] and myosin light chain 1 [99], filamin C [69,100] and titin [69,77] were also demonstrated to be CAPN3 substrates. All of these proteins are constituents of the sarcomere, serving either a structural role (nebulin, filamin and titin) or, as part of the contractile apparatus, a role in muscle contraction (MLC1); each of these proteins is part of a highly organized and enmeshed tight structure of the sarcomere.

Let us first examine circumstances of muscle function in order to consider why and under what circumstances CAPN3 would be called to cleave these proteins. A muscle cell can go through enormous extremes, from total rest to maximal contraction like in a sprint, marathon or weight lifting (this is not true for cardiac muscles where extremes are not that far off and where mature fulllength CAPN3 is also absent). Thus, these high-molecular weight molecules constitute particularly sensitive targets for any damage or hydrolytic cleavage of their polypeptide chains, which could be induced by the contraction-extension cycles of a functioning muscle. Indeed, these large proteins are not immune to simple shear or damage, as they are per definition more vulnerable than others, simply as a consequence of size (e.g. assuming an average probability function of hydrolysis per amino acid unit). It is reasonable to hypothesize that if optimal muscle function could be compatible with shorter molecules, natural selection would have since long favored the emergence of such entities. Yet, this did not happen. Hence, if titin, nebulin and a few other giant polypeptides are there, it is because they are needed to ensure efficient mechanotransduction and passive tension, despite their increased inherent vulnerability and risk for accumulation of breakdown products. The latter are likely to be generated with regular muscle use or disuse, a trend that might even be further exacerbated when muscle force generation is high, such as during weight lifting (e.g. [98,101]). Such accidental cleavage can occur anywhere within the sarcomeric bundle, with two practical consequences: a local disorganization of the regular lattice structure and the presence of damaged proteins or fragments thereof. The accumulation of, the latter, if not removed or replaced, would lead to cellular toxicity and be progressively and incrementally disruptive, leading to a gradual further disorganization of the sarcomere structure, to sarcomere fragility and eventually to striated muscles losing their ability to contract. Sarcomere loss would be a gradual and incremental process over repeated periods of such muscle injuries, which may explain the progressive nature of the disease. This pathophysiological model is compatible, on one hand, with the observation that CAPN3 was shown to promote myofibrillar protein turnover during sarcomere remodeling in post-natal wild type muscle [29], and on the other, with reports of lobulated fibers and disorganized or disrupted myofibrillar structures seen in CAPN3deficient muscles $[3,42,75,77,102]$, which once past a certain threshold, may become the target of uncontrolled protease action and ultimately lead to sarcomere disassembly and muscular dystrophy. Findings of increased heat shock proteins in the insoluble fraction of CAPN3 knock-out muscles is consistent with an essential role for this protease in proper protein turnover.

Hence when a molecular damage occurs in myofibrils, it has to be rapidly repaired and myofibrillar protein turnover must take place. This involves proteolytic action and degradation followed by protein replacement. Given its highly structured lattice organization, sensing and reaching the sites of damage may not be an easy task for any agent external to the sarcomere. Considering (1) the greatly reduced diffusion rate within the sarcomere $[96,97]$, and that (2) tightly packed sarcomeres are inherently relatively resistant to proteolytic action [103], (3) that the latter activity ought to be precisely localized and fine tuned, this may imply the necessity for a local loosening of the structure and an appropri- 
ate labeling of the material to be disposed of. Herein we propose that CAPN3 seems well suited to carry out the proposed task: because of its characteristic features, the presence of mature fulllength CAPN3 may represent the functional compromise between the necessity for both giant proteins and eventually the dispersal or removal of toxic breakdown products. First of all, full-length CAPN3 is a proximity agent. Obviously it cannot be too aggressive and do more damage than is needed to repair normal functional wear and tear. This may be one reason why it is so innately unstable once in a free form. Obviously it would be better if it were not calcium-activated, which is the case, as calcium may not always be around at the exact spot where it is needed, and furthermore calcium influxes may trigger much more general and less controlled reactions, resulting in large additional pleiotropically negative side effects that the cell must avoid. Repair proteolysis has to be very localized. By anchoring CAPN3 to titin, dispersed within the sarcomere, one also ensures that CAPN3 release can occur in a timely fashion at or near the site of molecular injury.

These CAPN3 substrates and their digestion products are toxic and hence cannot be allowed to accumulate. The resulting fragments need to be removed from within the sarcomere and eliminated, which is not an easy task due to the tightly bound structure of the sarcomere. This removal can be facilitated if CAPN3 activity results in a transient local loosening of the sarcomere bundle, for instance if it also removed neighboring intact associated proteins, allowing diffusion of these small cleavage products away from the sarcomere or by allowing additional proteases to act on these substrates. CAPN3's cleavage products can be marked for further proteasome-mediated degradation (possibly by the N-end rule pathway (which selectively degrades proteins that have a basic or large hydrophobic N-terminal residues); [104]), by E3 ligases such as Trim32 or MURF-1, which (in the case of MURF-1) is also located in the sarcomere. E3 ligases are the final enzymes in the process of adding polyubiquitin chains to protein substrates, targeting them to the proteasome. CAPN3 would thus not only act upstream of the ubiquitin pathway but be necessary for its ability to eliminate sarcomeric proteins, as indicated by the reported failure to activate high-molecular weight Ub-protein conjugates during recovery upon muscle disuse in CAPN3 knock-out as compared to wild type mice [29]. This postulated link with the proteasome is compatible with the fact that the E3 ubiquitin ligase Trim32, when mutated can also give rise to a LGMD [23]. A cartoon diagram is provided (Fig. 2) to demonstrate how CAPN3 might function in the sarcomere in conjunction with E3 ubiquitous ligases.

The nice property of this model is that it leads to a specific and limited chain of events. As hypothesized, CAPN3-mediated proteolysis may also destabilize the complex locally and allow other proteases to enter near the site of injury. In the absence of CAPN3 such facilitation processes do not occur, preventing the needed repairing step. Now, suppose there is a threshold for the accumulated amount of incurred damage that needs to be passed before functional physiological consequences become visible. Thus initially, local unrepaired damage will leave a molecular unhealable scar, without further major visible consequences, i.e., the rest of the structure remains unaltered. Then, as more damage is inflicted, the number of such scars increases, passing a critical threshold wherein there is enough accumulated damage to destabilize part of the myofibril, that results in the rapidly observable destruction of the (even disorganized) scaffold. These processes could eventually trigger inflammation, such as that observed in the eosinophilic myositis, also due to CAPN3 mutations. All of these events, if further aggravated, will lead to muscle pathology. Thus, repeated injuries will eventually lead to accumulation of protein debris, and result in destruction and dystrophy.

CAPN3 has indeed all the required attributes to be this "first repair factor", sitting and waiting just like water hoses along the streets, waiting for fire... that does eventually come. There is apparently never a pool of activated CAPN3, or if any, only in highly localized foci. But it can be rapidly activated; its stability (expressed as half-life in vitro) is such that if needed it has the capacity, through autolytic degradation, to turn over rapidly (hence it makes much sense that its preferred substrate is itself $[67,68,70]$, even in trans); it works at endogenous calcium concentrations and thus needs no other activation trigger to start functioning than to be released from its trap near the site where it has to enter into limited and controlled action. CAPN3 would thus be the first agent involved in the repair and maintenance of sarcomere integrity, acting possibly upstream of other factors such as E3 ligases (e.g. Trim32, MURF), that may target the CAPN3 digestion products for controlled elimination by the proteasome [29], and possibly the ubiquitous calpains. The recent reports of the association of eosinophilic myositis with mature full-length CAPN3 mutations [105] are thus also coherent with the model discussed herein. Noteworthy also is the reported accumulation of insoluble protein aggregates in skeletal muscles of CAPN3 knockout mice [29].

Binding to titin need not to be tight or stable. As shown by Ojima et al. [81], CAPN3 can hop during the contraction extension cycle from one titin site to an adjacent one (presumably in cis, i.e., along the same titin molecule, but possibly also in trans on an adjacent molecule). Thus, adding but a tiny twist to Ojima et al.'s suggestion that CAPN3 senses myofibrillar stretch, we would propose that it simultaneously senses also its integrity: the propensity of CAPN3 to switch localization back and forth from the N2A to the M-line and possibly jump to adjacent sites and/or molecules during sarcomere overextension and contraction may facilitate its role in sensing sarcomere integrity and hence in maintenance of a functional sarcomere structure. When the structure is not properly in phase, CAPN3 could self-activate and start its repair action. And when some CAPN3 has been lost through autolysis, local CAPN3 distribution can likewise be rapidly compensated through displacement from neighboring sites.

Whatever the exact role of CAPN3/p94, it is important to determine the nature of the triggers that make it come loose. Simple titin damage could be the signal required for CAPN3 release and action or that it acts as a ruler sensing proper phasing of the sarcomeric subunits. It is possible that titin per se is not sufficient as a signal and that it requires other partners or agents or some posttranslational modifications, but for sure, titin seems to play a key if not the major role. Titinopathies can also be reviewed under this new model, as they could affect calpains 3's binding, release and availability. Obviously all the above is also compatible with additional functions for mature full-length CAPN3, such as helping to control the regular assembly and maintenance of this ladder-like, array of proteins surrounding or bound to titin. It is clear that CAPN3 can exist in discreet areas of the cell such as the nucleus or plasma membrane, and its cellular function at each of these sites will need to be elucidated. It is tempting to speculate that in these cellular compartments too, CAPN3 may be involved in proper maintenance and repair.

\section{Conclusions}

Genetic evidence points for a major causal role of the mature full-length CAPN3 in LGMD2A and we hypothesize that it is essentially a reactive one to repair usage-induced erosion, hence preserving myofibril integrity and function. Interestingly, an analogous proposition was made for another LGMD2 gene, dysferlin, involving in this case membrane repair functions [106], with the possible participation of AHNAK and CAPN3 [94].

Biochemical and immunohistochemical evidence suggest that mature full-length CAPN3's role is mostly confined to the sarcomere. CAPN3 is embedded there, inside this structure, like a Trojan 


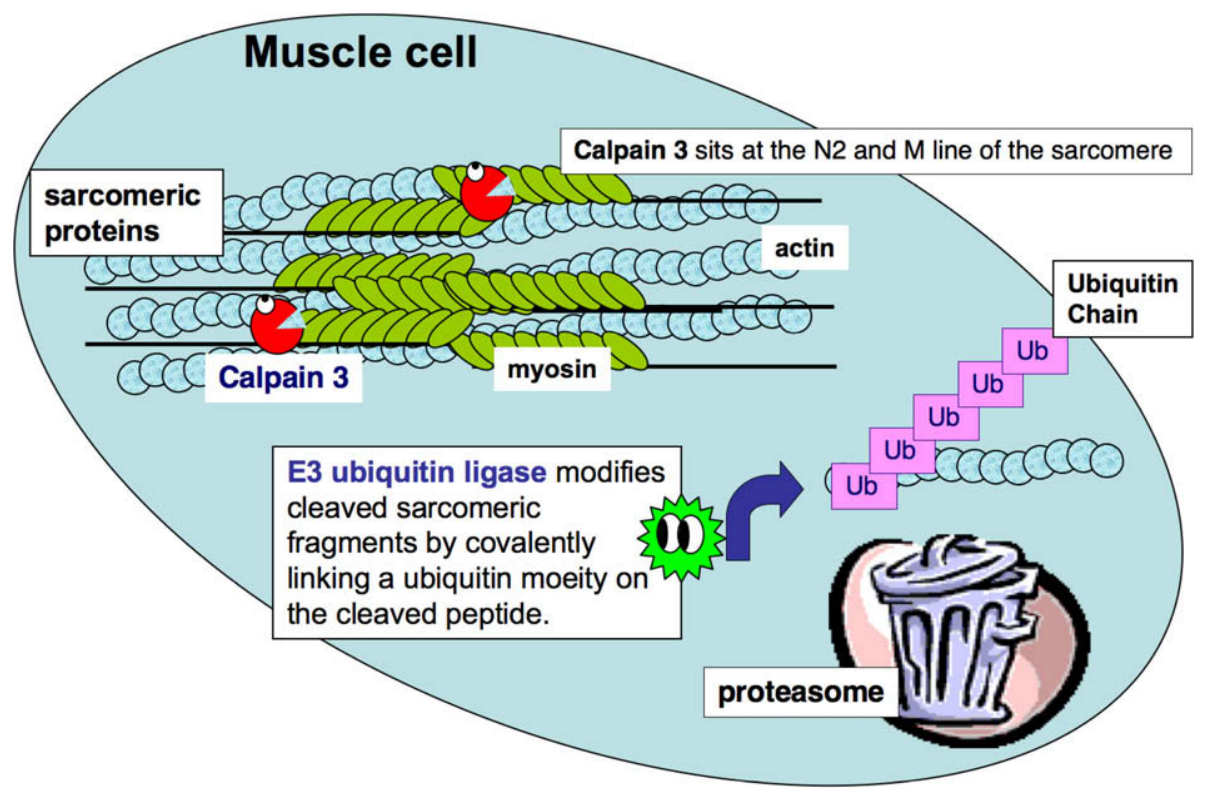

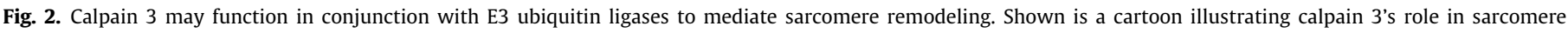

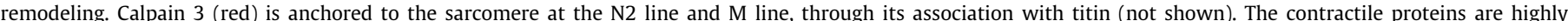

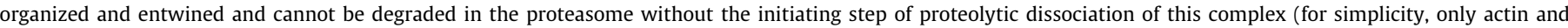

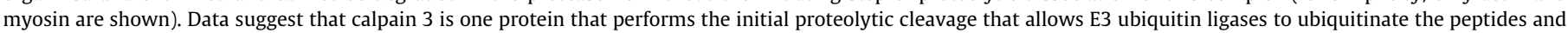
target them for degradation in the proteasome (see [29])

horse. To prevent it from acting in an unrestrained manner, it is bound and sequestered by titin. Once released, it will start acting, hydrolyze its substrates, the cytoskeletal and myofibrillar debris, contribute to relaxation and destabilization of the structure locally (beyond the effect of the mechanical damage), loosen the residual sarcomeric constituents, thereby facilitating removal of the debris and their access to other cellular proteases. To prevent it from being a Trojan horse, activated free mature full-length CAPN3 has also a very short lifetime, and it self digests before it can do any uncontrolled harm.

One testable prediction of the proposed functional model, is that any mutation abolishing full-length (IS1-containing) CAPN3's autoproteolytic lability yet preserving its normal alloproteolytic activity (just like wild type mature full-length CAPN3), if it can be generated, will be dominant and lead to a severe phenotype, like the transgenic mice expressing IS1- or IS2-less CAPN3 isoforms [87], possibly incompatible with life even in utero.

All the above does not imply that CAPN3 has no direct role in other cellular compartments in the cytosol or nucleus. Its nuclear role (or perhaps that of one of its autolytic degradation products) may be to induce the repair processes to replace the damaged and missing sarcomeric elements or regulate transcription factors $[69,90]$. But whatever the additional location it has to act in, it will be imperative to control its relatively rapid turnover rate and inhibit its unwanted effects. These activities are definitely not incompatible with the proposal as gatekeeper of sarcomere functional integrity. But it will be important to identify what stabilizes CAPN3 in the nucleus or other cellular compartments. The road towards the unraveling of the calpain mystery is long and fascinating.

\section{Acknowledgments}

We dedicate this Review to the memory of our dear friend and colleague, Dr. Darrel Goll. Darrel was the father of the calpain field who continued to make important scientific contributions and enrich our knowledge on calpains until his passing in the summer of 2008. Darrel was a devoted teacher, mentor and academician, keen to inspire and assist anyone who asked and always with a smile on his face. He will be greatly missed.

Both J.S.B. and M.S. thank our many laboratory members, direct colleagues and collaborators over the many years involved in this fascinating research area, as well as the larger calpain family and its regular encounters at the FASEB meetings. We thank Elena Kudryashova for generating the cartoon figure, Lorenzo Cerutti for his help with the bioinformatic analyses and to Irina Kramerova for enlightened discussion and hard work generating data over the years. We also thank Drs. P.L. Davies, D. Goll, E.C. Jackson, J.L. Mandel, Y. Ono and H. Sorimachi for constructive comments on this manuscript and Dr. Ernesto Carafoli for the image "conveyor of doom". J.S.B. is supported by a grant from the Swiss National Science Foundation (Grant 310000-112552/1) and the University of Lausanne, Switzerland and MJS by the NIH (NIAMS), the Muscular Dystrophy Association of America, the Parent Project for Muscular Dystrophy and the Jarvis Foundation for research on LGMD2A.

\section{References}

[1] Fardeau M, Eymard B, Mignard C, Tome FM, Richard I, Beckmann JS. Chromosome 15-linked limb-girdle muscular dystrophy: clinical phenotypes in Reunion Island and French metropolitan communities. Neuromuscul Disord 1996;6:447-53

[2] Fardeau M, Hillaire D, Mignard C, et al. Juvenile limb-girdle muscular dystrophy. Clinical, histopathological and genetic data from a small community living in the Reunion Island. Brain 1996;119(Pt. 1):295-308.

[3] Kaplan JC, Beckmann JS, Fardeau M. Limb girdle muscular dystrophies. In: Karpati G, Hilton-Jones D, Griggs RC, editors. Disorders of voluntary muscles. London: Cambridge University Press; 2001.

[4] Straub V, Bushby K. The childhood limb-girdle muscular dystrophies. Semin Pediatr Neurol 2006;13:104-14.

[5] Erb W. About the "juvenile form" of progressive muscle atrophy and its correlation to the so-called muscle pseudohypertrophy (Ueber die "juvenile Form" der progressiven Muskelatrophie und ihre Beziehungen zur sogenannten Pseudohypertrophie der Muskeln). Deutsch Archiv Klin Med 1884;34:467-519.

[6] Walton JN, Nattrass FJ. On the classification, natural history and treatment of the myopathies. Brain 1954;77:169-231.

[7] Brooke MH. A clinician's view of neuromuscular diseases. Baltimore: Williams \& Wilkins; 1986.

[8] Walton JN. Disorders of voluntary muscle. 5th ed. London: Churchill Livingstone; 1988 
[9] Gardner-Medwin D, Walton J. The muscular dystrophies. In: Walton J, Karpati G, Hilton-James D, editors. Disorders of voluntary muscles. Edinburgh: Churchill Livingstone; 1994.

[10] Chung CS, Morton NE. Discrimination of genetic entities in muscular dystrophy. Am J Hum Genet 1959;11:339-59.

[11] Jackson CE, Carey JH. Progressive muscular dystrophy: autosomal recessive type. Pediatrics 1961;28:77-84.

[12] Chutkow JG, Heffner Jr RR, Kramer AA, Edwards JA. Adult-onset autosomal dominant limb-girdle muscular dystrophy. Ann Neurol 1986;20:240-8.

[13] Gilchrist JM, Pericak-Vance M, Silverman L, Roses AD. Clinical and genetic investigation in autosomal dominant limb-girdle muscular dystrophy. Neurology 1988;38:5-9.

[14] Beckmann JS, Richard I, Hillaire D, et al. A gene for limb-girdle muscular dystrophy maps to chromosome 15 by linkage. C R Acad Sci III 1991;312:141-8.

[15] Beckmann JS, Brown RH, Muntoni F, Urtizberea A, Bonnemann C, Bushby KM. 66th/67th ENMC sponsored international workshop: the limb-girdle muscular dystrophies, 26-28 March 1999, Naarden, The Netherlands. Neuromuscul Disord 1999;9:436-45.

[16] Bushby KM, Beckmann JS. The limb-girdle muscular dystrophies - proposal for a new nomenclature. Neuromuscul Disord 1995;5:337-43.

[17] Bushby KM, Beckmann JS. The 105th ENMC sponsored workshop: pathogenesis in the non-sarcoglycan limb-girdle muscular dystrophies, Naarden, April 12-14, 2002. Neuromuscul Disord 2003;13:80-90.

[18] Beckmann JS, Bushby KM. Advances in the molecular genetics of the limbgirdle type of autosomal recessive progressive muscular dystrophy. Curr Opin Neurol 1996;9:389-93.

[19] Murachi T, Tanaka K, Hatanaka M, Murakami T. Intracellular $\mathrm{Ca}^{2+}$-dependent protease (calpain) and its high-molecular-weight endogenous inhibitor (calpastatin). Adv Enzyme Regul 1980;19:407-24.

[20] Sorimachi H, Imajoh-Ohmi S, Emori Y, et al. Molecular cloning of a novel mammalian calcium-dependent protease distinct from both $\mathrm{m}$ - and mutypes. Specific expression of the mRNA in skeletal muscle. J Biol Chem 1989;264:20106-11.

[21] Ono Y, Hayashi C, Doi N, et al. Comprehensive survey of p94/calpain 3 substrates by comparative proteomics - possible regulation of protein synthesis by p94. Biotechnol J 2007;2:565-76.

[22] Kawabata Y, Hata S, Ono Y, et al. Newly identified exons encoding novel variants of p94/calpain 3 are expressed ubiquitously and overlap the alphaglucosidase $C$ gene. FEBS Lett 2003;555:623-30.

[23] Frosk P, Weiler T, Nylen E, et al. Limb-girdle muscular dystrophy type $2 \mathrm{H}$ associated with mutation in TRIM32, a putative E3-ubiquitin-ligase gene. Am J Hum Genet 2002;70:663-72.

[24] Brockington M, Yuva Y, Prandini P, et al. Mutations in the fukutin-related protein gene (FKRP) identify limb girdle muscular dystrophy 2I as a milder allelic variant of congenital muscular dystrophy MDC1C. Hum Mol Genet 2001;10:2851-9.

[25] Godfrey C, Clement E, Mein R, et al. Refining genotype phenotype correlations in muscular dystrophies with defective glycosylation of dystroglycan. Brain 2007; $130: 2725-35$

[26] Murakami T, Hayashi YK, Noguchi S, et al. Fukutin gene mutations cause dilated cardiomyopathy with minimal muscle weakness. Ann Neurol 2006;60:597-602.

[27] Balci B, Uyanik G, Dincer P, et al. An autosomal recessive limb girdle muscular dystrophy (LGMD2) with mild mental retardation is allelic to WalkerWarburg syndrome (WWS) caused by a mutation in the POMT1 gene. Neuromuscul Disord 2005;15:271-5.

[28] Daniele N, Richard I, Bartoli M. Ins and outs of therapy in limb girdle muscular dystrophies. Int J Biochem Cell Biol 2007;39:1608-24.

[29] Kramerova I, Kudryashova E, Venkatraman G, Spencer MJ. Calpain 3 participates in sarcomere remodeling by acting upstream of the ubiquitinproteasome pathway. Hum Mol Genet 2005;14:2125-34.

[30] Duguez S, Bartoli M, Richard I. Calpain 3: a key regulator of the sarcomere? FEBS J 2006;273:3427-36.

[31] Chen YW, Gregory CM, Scarborough MT, Shi R, Walter GA, Vandenborne K. Transcriptional pathways associated with skeletal muscle disuse atrophy in humans. Physiol Genomics 2007.

[32] Emery AE. Population frequencies of inherited neuromuscular diseases - a world survey. Neuromuscul Disord 1991;1:19-29.

[33] Nigro V. Molecular bases of autosomal recessive limb-girdle muscular dystrophies. Acta Myol 2003;22:35-42.

[34] Piluso G, Politano L, Aurino S, et al. Extensive scanning of the calpain-3 gene broadens the spectrum of LGMD2A phenotypes. J Med Genet 2005;42:686-93.

[35] Urtasun M, Saenz A, Roudaut C, et al. Limb-girdle muscular dystrophy in Guipuzcoa (Basque Country, Spain). Brain 1998;121(Pt. 9):1735-47.

[36] Yates JR, Emery AE. A population study of adult onset limb-girdle muscular dystrophy. J Med Genet 1985;22:250-7.

[37] Fanin M, Nascimbeni AC, Fulizio L, Angelini C. The frequency of limb girdle muscular dystrophy $2 \mathrm{~A}$ in northeastern Italy. Neuromuscul Disord 2005;15:218-24.

[38] Passos-Bueno MR, Moreira ES, Marie SK, et al. Main clinical features of the three mapped autosomal recessive limb-girdle muscular dystrophies and estimated proportion of each form in 13 Brazilian families. J Med Genet 1996;33:97-102.
[39] Kramerova I, Beckmann JS, Spencer MJ. Molecular and cellular basis of calpainopathy (limb girdle muscular dystrophy type 2A). Biochim Biophys Acta 2007; 1772:128-44

[40] Richard I, Broux O, Allamand V, et al. Mutations in the proteolytic enzyme calpain 3 cause limb-girdle muscular dystrophy type 2A. Cell 1995;81: 27-40.

[41] Richard I, Roudaut C, Saenz A, et al. Calpainopathy - a survey of mutations and polymorphisms. Am J Hum Genet 1999;64:1524-40.

[42] Fanin M, Nascimbeni AC, Fulizio L, Trevisan CP, Meznaric-Petrusa M, Angelin C. Loss of calpain-3 autocatalytic activity in LGMD2A patients with normal protein expression. Am J Pathol 2003;163:1929-36.

[43] Ono Y, Shimada $\mathrm{H}$, Sorimachi $\mathrm{H}$, et al. Functional defects of a muscle-specific calpain, p94, caused by mutations associated with limb-girdle muscular dystrophy type 2A. J Biol Chem 1998;273:17073-8.

[44] Fanin M, Nascimbeni AC, Angelini C. Screening of calpain-3 autolytic activity in LGMD muscle: a functional map of CAPN3 gene mutations. J Med Genet 2007;44:38-43

[45] Jenne DE, Kley RA, Vorgerd M, et al. Limb girdle muscular dystrophy in a sibling pair with a homozygous Ser606Leu mutation in the alternatively spliced IS2 region of calpain 3. Biol Chem 2005;386:61-7.

[46] Jia Z, Petrounevitch V, Wong A, et al. Mutations in calpain 3 associated with limb girdle muscular dystrophy: analysis by molecular modeling and by mutation in m-calpain. Biophys J 2001:80:2590-6.

[47] Milic A, Daniele N, Lochmuller H, et al. A third of LGMD2A biopsies have normal calpain 3 proteolytic activity as determined by an in vitro assay. Neuromuscul Disord 2007;17:148-56.

[48] De Tullio R, Stifanese R, Salamino F, Pontremoli S, Melloni E. Characterization of a new p94-like calpain form in human lymphocytes. Biochem J 2003;375:689-96.

[49] Herasse M, Ono Y, Fougerousse $F$, et al. Expression and functional characteristics of calpain 3 isoforms generated through tissue-specific transcriptional and posttranscriptional events. Mol Cell Bio 1999;19:4047-55.

[50] Ma H, Fukiage C, Azuma M, Shearer TR. Cloning and expression of mRNA for calpain Lp82 from rat lens: splice variant of p94. Invest Ophthalmol Vis Sci 1998;39:454-61.

[51] Fougerousse F, Bullen P, Herasse M, et al. Human-mouse differences in the embryonic expression patterns of developmental control genes and disease genes. Hum Mol Genet 2000;9:165-73.

[52] Ma H, Shih M, Fukiage $C$, et al. Influence of specific regions in Lp82 calpain on protein stability, activity, and localization within lens. Invest Ophthalmol Vis Sci 2000;41:4232-9.

[53] Ma H, Shih M, Hata I, Fukiage C, Azuma M, Shearer TR. Protein for Lp82 calpain is expressed and enzymatically active in young rat lens. Exp Eye Res 1998;67:221-9.

[54] Konig N, Raynaud F, Feane H, et al. Calpain 3 is expressed in astrocytes of rat and Microcebus brain. J Chem Neuroanat 2003;25:129-36.

[55] Welm AL, Timchenko NA, Ono Y, et al. C/EBPalpha is required for proteolytic cleavage of cyclin A by calpain 3 in myeloid precursor cells. J Biol Chem 2002;277:33848-56

[56] Fanin M, Fulizio L, Nascimbeni AC, et al. Molecular diagnosis in LGMD2A: mutation analysis or protein testing? Hum Mutat 2004;24:52-62.

[57] Croall DE, Ersfeld K. The calpains: modular designs and functional diversity. Genome Biol 2007;8:218.

[58] Goll DE, Thompson VF, Li H, Wei W, Cong J. The calpain system. Physiol Rev 2003;83:731-801.

[59] Sorimachi H, Suzuki K. The structure of calpain. J Biochem (Tokyo) 2001:129:653-64.

[60] Carafoli E, Molinari M. Calpain: a protease in search of a function? Biochem Biophys Res Commun 1998;247:193-203.

[61] Goll DE, Neti G, Mares SW, Thompson VF. Myofibrillar protein turnover: the proteasome and the calpains. J Anim Sci 2007.

[62] Branca D, Gugliucci A, Bano D, Brini M, Carafoli E. Expression, partia purification and functional properties of the muscle-specific calpain isoform p94. Eur J Biochem 1999;265:839-46.

[63] Garcia Diaz BE, Gauthier S, Davies PL. $\mathrm{Ca}^{2+}$ dependency of calpain 3 (p94) activation. Biochemistry 2006;45:3714-22.

[64] Wendt A, Thompson VF, Goll DE. Interaction of calpastatin with calpain: review. Biol Chem 2004;385:465-72.

[65] Kinbara K, Ishiura S, Tomioka S, et al. Purification of native p94, a musclespecific calpain, and characterization of its autolysis. Biochem J 1998;335(Pt. 3):589-96.

[66] Ravulapalli R, Diaz BG, Campbell RL, Davies PL. Homodimerization of calpain 3 penta-EF-hand domain. Biochem J 2005;388:585-91.

[67] Ono Y, Kakinuma K, Torii F, et al. Possible regulation of the conventiona calpain system by skeletal muscle-specific calpain, p94/calpain 3. J Biol Chem 2004;279:2761-71.

[68] Ono Y, Torii F, Ojima K, et al. Suppressed disassembly of autolyzing p94/ CAPN3 by N2A connectin/titin in a genetic reporter system. J Biol Chem 2006;281:18519-31.

[69] Taveau M, Bourg N, Sillon G, Roudaut C, Bartoli M, Richard I. Calpain 3 is activated through autolysis within the active site and lyses sarcomeric and sarcolemmal components. Mol Cell Biol 2003;23:9127-35.

[70] Sorimachi H, Toyama-Sorimachi N, Saido TC, et al. Muscle-specific calpain, p94, is degraded by autolysis immediately after translation, resulting in disappearance from muscle. J Biol Chem 1993;268:10593-605. 
[71] Anderson LV, Davison K, Moss JA, et al. Characterization of monoclonal antibodies to calpain 3 and protein expression in muscle from patients with limb-girdle muscular dystrophy type 2A. Am J Pathol 1998;153:1169-79.

[72] Spencer MJ, Tidball JG, Anderson LV, et al. Absence of calpain 3 in a form of limb-girdle muscular dystrophy (LGMD2A). J Neurol Sci 1997;146:173-8.

[73] Granzier HL, Labeit S. Titin and its associated proteins: the third myofilament system of the sarcomere. Adv Protein Chem 2005;71:89-119.

[74] Lange S, Ehler E, Gautel M. From A to Z and back? Multicompartment proteins in the sarcomere. Trends Cell Biol 2006;16:11-8.

[75] Keira Y, Noguchi S, Minami N, Hayashi YK, Nishino I. Localization of calpain 3 in human skeletal muscle and its alteration in limb-girdle muscular dystrophy 2A muscle. J Biochem (Tokyo) 2003;133:659-64.

[76] Sorimachi H, Kinbara K, Kimura S, et al. Muscle-specific calpain, p94 responsible for limb girdle muscular dystrophy type $2 \mathrm{~A}$, associates with connectin through IS2, a p94-specific sequence. J Biol Chem 1995;270:31158-62.

[77] Kramerova I, Kudryashova E, Tidball JG, Spencer MJ. Null mutation of calpain 3 (p94) in mice causes abnormal sarcomere formation in vivo and in vitro. Hum Mol Genet 2004;13:1373-88.

[78] Ojima K, Ono Y, Hata S, Koyama S, Doi N, Sorimachi H. Possible functions of p94 in connectin-mediated signaling pathways in skeletal muscle cells. J Muscle Res Cell Motil 2005;26:409-17.

[79] Gregorio CC, Granzier H, Sorimachi H, Labeit S. Muscle assembly: a titanic achievement? Curr Opin Cell Biol 1999;11:18-25.

[80] Fougerousse F, Durand M, Suel L, et al. Expression of genes (CAPN3, SGCA, SGCB, and TTN) involved in progressive muscular dystrophies during early human development. Genomics 1998;48:145-56.

[81] Ojima K, Ono Y, Doi N, et al. Myogenic stage, sarcomere length, and protease activity modulate localization of muscle-specific calpain. J Biol Chem 2007;282:14493-504.

[82] Diaz BG, Moldoveanu T, Kuiper MJ, Campbell RL, Davies PL. Insertion sequence 1 of muscle-specific calpain, p94, acts as an internal propeptide. Biol Chem 2004;279:27656-66.

[83] Hackman P, Vihola A, Haravuori $\mathrm{H}$, et al. Tibial muscular dystrophy is a titinopathy caused by mutations in TTN, the gene encoding the giant skeletalmuscle protein titin. Am J Hum Genet 2002;71:492-500.

[84] Haravuori H, Vihola A, Straub V, et al. Secondary calpain3 deficiency in 2qlinked muscular dystrophy: titin is the candidate gene. Neurology 2001;56:869-77.

[85] Garvey SM, Rajan C, Lerner AP, Frankel WN, Cox GA. The muscular dystrophy with myositis (mdm) mouse mutation disrupts a skeletal muscle-specific domain of titin. Genomics 2002;79:146-9.

[86] Huebsch KA, Kudryashova E, Wooley CM, et al. Mdm muscular dystrophy: interactions with calpain 3 and a novel functional role for titin's N2A domain. Hum Mol Genet 2005;14:2801-11.

[87] Spencer MJ, Guyon JR, Sorimachi H, et al. Stable expression of calpain 3 from a muscle transgene in vivo: immature muscle in transgenic mice suggests role for calpain 3 in muscle maturation. Proc Natl Acad Sci USA 2002;99:8874-9

[88] Carmignac V, Salih MA, Quijano-Roy S, et al. C-terminal titin deletions cause a novel early-onset myopathy with fatal cardiomyopathy. Ann Neurol 2007;61:340-51.

[89] Beckmann JS. Genetic studies and molecular structures: the dystrophin associated complex. Hum Mol Genet 1996;5:865-7.
[90] Baghdiguian S, Martin M, Richard I, et al. Calpain 3 deficiency is associated with myonuclear apoptosis and profound perturbation of the IkappaB alpha/ NF-kappaB pathway in limb-girdle muscular dystrophy type $2 \mathrm{~A}$. Nat Med 1999;5:503-11.

[91] Kramerova I, Kudryashova E, Wu B, Spencer MJ. Regulation of the Mcadherin-beta-catenin complex by calpain 3 during terminal stages of myogenic differentiation. Mol Cell Biol 2006;26:8437-47.

[92] Huang Y, Verheesen P, Roussis A, et al. Protein studies in dysferlinopathy patients using llama-derived antibody fragments selected by phage display. Eur J Hum Genet 2005;13:721-30.

[93] Anderson LV, Harrison RM, Pogue R, et al. Secondary reduction in calpain 3 expression in patients with limb girdle muscular dystrophy type $2 \mathrm{~B}$ and Miyoshi myopathy (primary dysferlinopathies). Neuromuscul Disord 2000;10:553-9.

[94] Huang Y, de Morree A, van Remoortere A, et al. Calpain 3 is a modulator of the dysferlin protein complex in skeletal muscle. Hum Mol Genet 2008; $17: 1855-66$

[95] Richard I, Roudaut C, Marchand S, et al. Loss of calpain 3 proteolytic activity leads to muscular dystrophy and to apoptosis-associated IkappaBalpha/ nuclear factor kappaB pathway perturbation in mice. J Cell Biol 2000;151:1583-90.

[96] Papadopoulos S, Endeward V, Revesz-Walker B, Jurgens KD, Gros G. Radial and longitudinal diffusion of myoglobin in single living heart and skeletal muscle cells. Proc Natl Acad Sci USA 2001;98:5904-9.

[97] Papadopoulos S, Jurgens KD, Gros G. Protein diffusion in living skeletal muscle fibers: dependence on protein size, fiber type, and contraction. Biophys J 2000;79:2084-94.

[98] Feasson L, Stockholm D, Freyssenet D, et al. Molecular adaptations of neuromuscular disease-associated proteins in response to eccentric exercise in human skeletal muscle. J Physiol 2002;543:297-306.

[99] Cohen N, Kudryashova E, Kramerova I, et al. Identification of putative in vivo substrates of calpain 3 by comparative proteomics of overexpressing transgenic and nontransgenic mice. Proteomics 2006;6:6075-84.

[100] Guyon JR, Kudryashova E, Potts A, et al. Calpain 3 cleaves filamin C and regulates its ability to interact with gamma- and delta-sarcoglycans. Muscle Nerve 2003;28:472-83.

[101] Murphy RM, Goodman CA, McKenna MJ, Bennie J, Leikis M, Lamb GD. Calpain-3 is autolyzed and hence activated in human skeletal muscle $24 \mathrm{~h}$ following a single bout of eccentric exercise. J Appl Physiol 2007;103: 926-31.

[102] Chae J, Minami N, Jin Y, et al. Calpain 3 gene mutations: genetic and clinicopathologic findings in limb-girdle muscular dystrophy. Neuromuscul Disord 2001;11:547-55.

[103] Solomon V, Goldberg AL. Importance of the ATP-ubiquitin-proteasome pathway in the degradation of soluble and myofibrillar proteins in rabbit muscle extracts. J Biol Chem 1996;271:26690-7.

[104] Solomon V, Baracos V, Sarraf P, Goldberg AL. Rates of ubiquitin conjugation increase when muscles atrophy, largely through activation of the $\mathrm{N}$-end rule pathway. Proc Natl Acad Sci USA 1998;95:12602-7.

[105] Krahn M, Lopez de Munain A, Streichenberger N, et al. CAPN3 mutations in patients with idiopathic eosinophilic myositis. Ann Neurol 2006;59: 905-11.

[106] Bansal D, Miyake K, Vogel SS, et al. Defective membrane repair in dysferlindeficient muscular dystrophy. Nature 2003;423:168-72. 